Trends Cogn Sci. 2009 December ; 13(12): 524-531. doi:10.1016/j.tics.2009.10.002.

\title{
The Neural Correlates of Race
}

\author{
Tiffany A. Ito and \\ University of Colorado, Department of Psychology and Neuroscience, 345 UCB, Boulder, CO \\ 80309-0345 \\ Bruce D. Bartholow \\ Department of Psychological Sciences, 10 McAlester Hall, University of Missouri, Columbia, MO \\ 65211
}

\begin{abstract}
Behavioral analyses are a natural choice for understanding the wide-ranging behavioral consequences of racial stereotyping and prejudice. However, neuroimaging and electrophysiological research has recently considered the neural mechanisms that underlie racial categorization and the activation and application of racial stereotypes and prejudice, revealing exciting new insights. Work reviewed here points to the importance of neural structures previously associated with face processing, semantic knowledge activation, evaluation, and self-regulatory behavioral control, allowing for the specification of a neural model of race processing. We show how research on the neural correlates of race can serve to link otherwise disparate lines of evidence on the neural underpinnings of a broad array of social-cognitive phenomena, and consider implications for effecting change in race relations.
\end{abstract}

\section{New Frontiers in the Study of Race and Social Cognition}

Brain imaging and electrophysiological methods have emerged as important new tools for scholars of race. In particular, research using functional brain imaging (e.g., fMRI) and electrocortical responses (EEG/ERPs) is providing unparalleled access to how race is processed in the brain, as well as new insights on how race influences perceptions and behaviors (see Box 1 for the longer history of studying race relations with physiological measures). Several recent models explicate neural structures involved in particular aspects of social cognition, such as judging mental states $\left[1,{ }^{2}\right]$, perceiving faces [3], and activating attitudes [4]. Although such models are critical for understanding specific social-cognitive constructs, it is important to recognize that in vivo social perception draws on multiple, overlapping social cognitive processes. Race perception permits examination of how these and related systems interact to inform judgments and behaviors. Here we review representative recent research investigating the neural systems associated with race processing. On the basis of this work, we sketch an initial model of the neural correlates of race that can serve as a basis for future research aimed at understanding the interacting systems involved in race processing and its downstream cognitive, affective and behavioral consequences.

Corresponding author: Ito, T.A. (tiffany.ito@ colorado.edu).

Publisher's Disclaimer: This is a PDF file of an unedited manuscript that has been accepted for publication. As a service to our customers we are providing this early version of the manuscript. The manuscript will undergo copyediting, typesetting, and review of the resulting proof before it is published in its final citable form. Please note that during the production process errors may be discovered which could affect the content, and all legal disclaimers that apply to the journal pertain. 


\section{Box 1}

\section{A Brief History of Psychophysiological Responses to Race}

While contemporary social neuroscience research has emphasized neural mechanisms of racial perception, the roots of this more recent work can be traced to numerous early studies that employed peripheral physiological measures. At the time of these initial investigations, researchers were motivated to understand whether post-World War II changes in cultural attitudes that made Caucasian research participants increasingly reluctant to verbally express racial prejudice reflected a true lack of racial antipathy. Physiological measures were used in this endeavor as a way to more covertly assess reactions to race.

In one of the first known efforts of this kind, Rankin and Campbell [49] measured electrodermal responses (i.e., skin conductance) from Caucasian participants as they interacted with either a Caucasian or a Black experimenter. They found significantly larger responses to the Black than to the Caucasian experimenter, suggesting heightened anxiety responses-attributed to more negative attitudes-to Blacks (see also [50]). Others reported similar findings among Caucasian participants for anticipated contact with Blacks [51], as well as for simple visual depictions of Black targets $\left[52,{ }^{53}\right]$.

In addition to electrodermal responses, researchers have used electromyographic (EMG) measures to covertly assess reactions to race. EMG provides a sensitive measure of even very small movements of facial muscles associated with negative (i.e., frowning) and positive (i.e., smiling) reactions to stimuli (see [54]), including targets varying by race. In a seminal study of this kind, Vanman, Paul, Ito, and Miller [55] recorded EMG as Caucasian participants imagined cooperative work experiences with Black and Caucasian partners. EMG responses indicated negative covert reactions to Black targets (i.e., heightened activation of the corrugator supercilii muscles, associated with expression of negative affect) despite very positive overt ratings of the Black targets (see also [56]).

Studies using peripheral physiological measures were instrumental in highlighting the relevance of bodily reactions to understanding complex psychological processes (for an indepth review, see [57]), and thereby laid the foundation for the more recent investigations reviewed in the main text. Contemporary researchers in the burgeoning field of social neuroscience owe a considerable debt to these early pioneers and the knowledge their work produced. Moreover, research in this tradition remains active, as scientists continue to make important theoretical advances using a broad range of psychophysiological measures, including cardiovascular and neuroendocrine responses (e.g. $\left.\left[58,{ }^{59}\right]\right)$.

\section{Race Perception, Categorization, and the Putative Face Processing Network}

Racial categorization can occur based on facial features, which means that race perception often begins with the perception of a face. Consequently, this review begins by considering research on the neural structures supporting face processing. Although work on face perception often focused on mechanisms that differentiate faces from non-faces, and on how we retrieve personal identity [e.g., ${ }^{3}$, growing research shows that even very basic aspects of face perception are affected by race, and that sensitivity to race occurs in a very fast and seemingly automatic fashion.

\section{Fusiform gyrus and posterior cingulate cortex (PCC)}

Race effects have been observed in two brain areas traditionally associated with face perception, the lateral fusiform gyrus and PCC, with greater activity to racial ingroup than outgroup members in both areas $\left[5^{8}\right]$ (but see Box 2 for more equivocal results from electrocortical studies of fusiform responses). Lateral fusiform activity has been linked to 
encoding of the visual appearance of the face. Modulation of this process by race could reflect greater experience with racial ingroup members, as other research shows an increase in fusiform activity with expertise [9]. Motivation may also play a role, as suggested by a study in which Caucasian participants were told they had been randomly assigned to one of two competing, racially-diverse teams [10]. Participants first learned to recognize members of both teams, then viewed pictures of team members' faces. Activity in bilateral fusiform gyrus was sensitive to team designation, showing greater activity to own than competing team members regardless of their race. Thus, the motivational significance conferred by status as a fellow team member modulated recruitment of face processing mechanisms; suggesting that prior race effects could reflect inherent motivations to more deeply attend to ingroup members. Regardless of the cause, race effects on fusiform activity have downstream consequences; greater activity in left fusiform to ingroup than outgroup faces also correlates with an ingroup memory advantage [5].

\section{Box 2}

\section{Race Effects in the N170 Electrocortical Index of Face Processing}

The N170 ERP component is a negative-going deflection maximal over lateral temporal areas that is larger to faces than non-faces [60]. Its sensitivity to race has been equivocal, with support obtained for three mutually exclusive hypotheses. First, the N170 has been argued to reflect structural face encoding, sensitive only to global features that differentiate faces from non-faces, but not to features of individual faces $\left[60,{ }^{61}\right]$. This perspective implies that race should not modulate N170 responses, a pattern obtained in several studies [62$\left.{ }^{64}\right]$.

Other research indicates that N170 amplitude is also increased to non-face stimuli with which participants are expert [65]. This suggests the N170 reflects a more general expertise mechanism sensitive to faces, about which humans are normally expert, and to other stimuli about which individuals are idiosyncratically expert. This perspective predicts that N170 amplitude should be greatest to racial ingroup members because perceivers typically have more experience interacting with ingroup members. This also converges with source localization data implicating the fusiform gyrus in the generation of the N170 [66], and the greater fusiform activity to racial ingroup faces observed in fMRI studies $\left[5,{ }^{6}\right]$. To date, though, only one study has obtained this N170 pattern [14].

Finally, N170 amplitude is increased by manipulation that disrupts the configural processing typically applied to faces [67]. Racial outgroup faces are often less familiar, and may be processed in a less configural manner [68]. Consequently, the prediction can be derived that N170s should be larger to racial outgroup faces, a pattern obtained in several studies [69${ }^{71}$ ].

These conflicting findings suggest that features other than simple physical/structural differences influence N170 responses to faces. One possibility is that motivational factors that make race more or less salient to perceivers modulate this neural response. Consistent with this view, all studies producing larger N170s to racial outgroups have made identity salient (e.g., by having participants detect when two consecutively presented faces match or trying to remember the faces) whereas none of the studies obtaining other patterns have. Given assumptions that the N170 reflects structural face encoding, and that perceivers typically process racial outgroup faces in a less configural shallower manner (e.g., as reflected in poorer memory for outgroup faces), tasks that require attention to identity may selectively increase recruitment of face processing mechanisms to racial outgroup members. However, further research is needed to clarify these seemingly contradictory patterns of N170 to racial outgroup and ingroup targets. 
PCC activity generally is enhanced during retrieval of information about familiar versus unfamiliar individuals [3]. However, enhanced PCC activity to ingroup faces has been obtained with faces that are unknown to the participants, suggesting that a more general sense of groupbased familiarity also affects PCC activity. In sum, work examining race effects on face processing both informs understanding of race categorization and suggests that motivational factors (e.g., those relevant to ingroup-outgroup distinctions) importantly influence recruitment of face processing mechanisms, thereby enhancing understanding of this fundamental component of social cognition.

\section{Electrocortical responses to race}

While fMRI has revealed neural structures affected by race during face processing, studies using event-related brain potentials (ERPs) have elucidated mechanistic aspects of race perception and categorization, such as its timecourse and malleability. In an initial investigation, participants viewed pictures of Black and Caucasian individuals (targets) while ERPs were recorded [11]. Modulations as a function of target race occurred as early as the N100 ERP component, peaking $122 \mathrm{~ms}$ after face onset. Race effects also were observed in the subsequent P200, N200, and P300 components (see Figure 1). Numerous subsequent investigations have replicated these findings $\left[12-{ }^{18}\right]$ (see Table 1). These components are generally sensitive to attentional and categorization processes [19], suggesting that the race effects reflect automatic encoding of and orienting toward racial category information. Importantly, sensitivity to race occurs whether participants are explicitly attending to race, attending to another social dimension (gender), or making person-based, individuating judgments [14], indicating that attention to race is fairly obligatory.

Just as face processing areas appear sensitive to ingroup-outgroup distinctions, effects of race on ERP components reflect distinctions between ingroup and outgroup members. Dickter and Bartholow [12] recorded ERPs as Black and Caucasian perceivers viewed pictures of Black and Caucasian targets in a gender categorization task. Results for Caucasian perceivers replicated previous work (e.g. $\left.\left[11,{ }^{14},{ }^{15}\right]\right)$, showing larger P200 amplitude to Black than to Caucasian targets and larger N200 amplitude to Caucasian than to Black targets. However, the pattern was reversed among Black perceivers, with larger P200s to Caucasian targets and larger N200s to Black targets. Willadsen-Jensen and Ito [17] reported similar findings with Asian participants. These results, coupled with the fMRI face processing data, suggest that neural differentiation of race operates at the level of broader social distinctions (ingroup vs. outgroup).

Of importance, neural signals reflecting early, spontaneous racial distinctions are related to subsequent race-biased responding. In one study, participants viewed pictures of Caucasian and Black men holding small objects, and had to quickly indicate via key press whether the object was a gun or something innocuous (e.g., cell phone) [20]. Participants whose P200 and N200 ERP responses indicated greater differentiation between Black and Caucasian targets showed more pronounced behavioral bias (i.e., faster responses to armed Blacks than armed Caucasians). Moreover, ERP effects mediated the relation between self-reported endorsement of stereotypes linking Blacks with threat and behavioral expression of racial bias. Thus, the ERP-behavior correlation in this study suggests a link between electrocortical sensitivity to race and application of activated racial stereotypes.

\section{Race Perception, Stereotype Activation, and Behavior}

Countless studies have shown that categorizing another's race (whether intentionally or not) spontaneously activates beliefs linked to their racial category (e.g. $\left[21,{ }^{22}\right]$ ). These beliefs (i.e., stereotypes) then influence reactions toward and judgments about the individual. To date, few studies have sought to identify brain areas associated with racial stereotyping (see Box 3) but ERPs have been used to investigate rapidly-unfolding neural responses to stereotype activation 
and violation. Bartholow and colleagues [23] recorded ERPs during a face priming task known to elicit stereotype activation. The P300 component was sensitive to stereotype violations, with counter-stereotypic associations eliciting larger amplitude and longer latency than stereotypeconsistent associations. The $\mathrm{P} 300$ has been linked to activity in the broadly-distributed locus coeruleus-norepinephrine system, which responds to motivationally-significant events [24]. Stereotype violations challenge existing semantic knowledge and thus are hypothesized to engage this system, reflecting either updating of existing content or a motivated attempt to resolve the inconsistent information.

\section{Box 3}

\section{Neural Structures Relevant for Understanding Stereotype Activation}

Although few studies to date have directly investigated the neural underpinnings of racial stereotype activation, consideration of work in related domains suggests brain areas that may be involve in racial stereotyping. Stereotypes can be considered analogous to other constructs stored in semantic memory, and therefore research investigating the neural structures supporting semantic memory is relevant to consider. Several studies have pointed to areas of the medial temporal lobes as important for semantic memory retrieval [47]. Additionally, considerable work implicates areas within PFC as important for both semantic retrieval and for cognitive control, and suggests that semantic knowledge is an important moderator of the extent to which control is implemented [see ${ }^{48}$ ]. As reviewed elsewhere in this article, control-related resources marshaled in PFC are important for regulation of stereotype-based responding. Thus, this work underscores the functional dependence between knowledge of stereotypes and the ability to control responses based on them.

This notion provides a context for interpreting the results of another recent study. investigating the neural correlates of gender stereotype activation and application [72]. Findings indicated an extensive area of right PFC that distinguished trials in which stereotypes were applied from those in which they were not applied. Moreover, activation in this region -- and only this region -- correlated with a behavioral measure of implicit gender stereotyping. These results suggest that stereotype application relies on cognitive processes generally underlying semantic knowledge about categories.

Finally, although not directly associated with stereotype activation, recent work implicates specific anterior temporal lobe regions and connections with MPFC in conceptual knowledge about people. A recent review [73] indicated that the superior temporal sulcus stores semantic representations of functional knowledge about people. Similarly, Mitchell and colleagues [74] reported that person knowledge (as opposed to object knowledge) was associated with activity in superior temporal cortex and MPFC.

Determining whether racial stereotyping is subserved by the same structures identified in these other lines of work awaits future research. However, given the structural similarity between stereotypes and semantic knowledge in general, the conceptual similarity between racial and gender stereotypes, and the consistency with which aspects of social judgment activate these regions, it is reasonable to predict that the superior temporal lobes and MPFC are strong candidates for structures involved in activation and implementation of racial stereotypes.

Subsequent research investigated spontaneous stereotype activation in a task that required no explicit trait inferences [25]. Participants categorized the race of centrally-presented Black and Caucasian faces (targets) flanked on 4 sides by trait words that were either stereotype-consistent (e.g., a Black face with "violent") or stereotype-inconsistent (e.g., a Black face with "safe") with respect to the target's race. Strictly speaking, the flanker words were task-irrelevant and 